\title{
WidSets: A Usability Study of Widget Sharing
}

\author{
Kristiina Karvonen ${ }^{1}$, Theofanis Kilinkaridis ${ }^{1}$, and Olli Immonen ${ }^{2}$ \\ ${ }^{1}$ Helsinki Institute for Information Technology HIIT, \\ Helsinki University of Technology \\ \{kristiina.karvonen, theofanis.kilinkaridis\}@hiit.fi \\ ${ }^{2}$ Nokia \\ olli.immonen@nokia.com
}

\begin{abstract}
In this paper, we describe a study conducted to better understand and improve the usability of the reputation elements on an online widget sharing site called WidSets. With a series of interviews and an online questionnaire with users of WidSets, we seek to understand the motivations and instigators of the actual usage, the level of understanding, and acceptance of current reputation elements. We analyse the impact of these factors on WidSets users' willingness to download and use the publicly available widgets. The outcomes show the visual elements of the user interface to predominate as the source for information for the decision to trust and download. However, the accuracy of the interpretation of these elements remains ambiguous. There also seems to be little awareness of the underlying security issues related to possible malicious widgets and developers.
\end{abstract}

Keywords: Widget, reputation, usability, user study, questionnaire, trust.

\section{Introduction}

Web widgets are PC or mobile micro-applications performing a dedicated task such as showing news headlines, a weather forecast or a Wikipedia article. Widgets are typically freeware distributed through widget repositories ${ }^{1}$. In some cases, downloading a widget may involve a risk of abuse of user's private information such as contacts and location. Widget repositories typically do not guarantee the quality or trustworthiness of all widgets. In WidSets, some widgets have been verified by the staff. Users need to make downloading decisions based on widget description, logo, developer history and data reflecting other users' satisfaction with the widget, such as number of downloads, rating and comments.

In this paper, we describe the results of a study regarding usability of the reputation elements on the WidSets site. The study was performed as an interview and a webbased questionnaire among current WidSets users $(n=38)$.

Our results indicate that while only a small part of the users find it difficult to use the web site or the mobile UI, the information related to trustworthiness and

\footnotetext{
${ }^{1}$ E. g. widsets.com, www.apple.com/downloads/dashboard, widgets.yahoo.com
} 
reputation is either poorly understood or underutilised. The most important elements to affect downloading decision were 1) the description, either 2a) rating (in web site) or 2b) number of users (in mobile UI), and 3) widget logo. The results suggest that visual appearance greatly influences users' decision making. Current users mostly trust the WidSets site while at the same time almost half consider the risk to be confronted by harmful content to be real.

\section{Background}

Reputation systems are used in internet services where users need to make trust decision concerning people they do not know beforehand. Reputation guides users' decision making. In eBay high reputation can lead to price premiums [1].

Online trust formation has many ingredients and has been widely researched on from various viewpoints, including technical, legal, social, psychological and philosophical [2]. For our work, the research on trust from a psychological viewpoint, as well as the social aspects of trust, especially work on collaboration and trust in the context of social networking seems most relevant.

WidSets is a combination of a service and a mobile Java MIDP2 client software. Users can browse the widget catalog with the phone client or with a PC on the WidSets web site (Fig. 1). "Picked" widgets are then downloaded and used in the client. Users can rate widgets, write comments and ask questions. Widgets can be developed and submitted to the site easily, e.g. by defining a logo and an RSS feed. More advanced widgets can be developed using the WidSets SDK and a scripting language, or web technologies (HTML, XHTML, JavaScript and CSS). Web technology widgets have features that are not available to regular web applications such as access to contacts, GPS and camera.
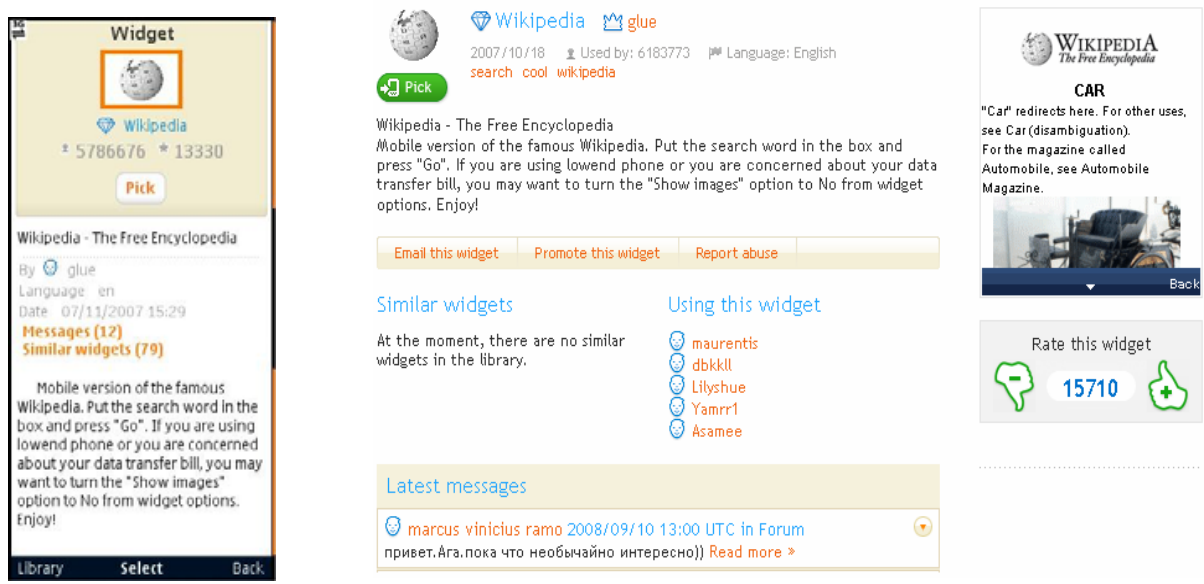

Fig. 1. Screenshot of WidSets mobile UI (on the left) and web site widsets.com (on the right) 


\section{The Study}

We began by interviewing current widget users to gain an initial understanding on how widgets are currently perceived and used. A web-based questionnaire ${ }^{2}$ was built on basis of the interview outcomes to gather feedback from more users. The questions in the questionnaire consisted of a modified version of the interview structure and topical areas. We had in all 38 users with 34 males and 3 females ${ }^{3} .58 \%$ had at least a bachelor's degree. All interviewees $(n=8)$ were Finnish and located in Finland, whereas six of the questionnaire respondents $(n=30)$ represented also various other nationalities. Most popular types of widgets were related to News, Weather, and Transportation. Over $75 \%$ users had been using the WidSets service for over a year. $10 \%$ had also developed and published their own widget, besides downloading.

Next, we analyse the outcomes in more detail.

Meaning of widgets. Users saw the service primarily as a mobile service, to make their mobile phone connected to the Internet: "It's a nice graphical user interface for accessing the Internet"; "I see it as a mobile service where you can get easily information"; "For me, it is a 'Web to Mobile' service".

Usage. The motivation for the usage varied: Some had come across the site at random on the Internet; some had learnt about the site on an online discussion board, when others had had their friends' recommendation of the site. Currently, the primary motivation behind widgets usage was to "get information", with "entertainment" coming second. The information-centricity was emphasized by other major motivations such as "work" and "organizing my day". Social pressure was one motivation: "[I use it] because my friends do".

User experience. Most users ranked both the website and the mobile user interface of the WidSets service as easy or very easy to use (58\% for website, $75 \%$ for mobile UI), very few finding it difficult to use ( $5 \%$ for website, $9 \%$ for mobile UI). Almost half of the users preferred the mobile UI over the website, $30 \%$ preferred the website and about $20 \%$ could not distinguish between the two. As the mobile usage dominates, it is natural that mobile UI becomes easier to use, through habituation. Another factor contributing to the experienced ease of use is probably the evident simplicity of the mobile UI, in comparison to the website

Reputation elements. To find out which reputation elements were used when deciding to download a particular widget, we showed users screenshots of the download phase for five widgets from both the WidSets website and mobile UI. Users were asked to select the three most and least important reputation elements.

For the website, users most looked at Description (27\%); Rating (21\%) and the Widget logo (12\%), while they ignored Date (2.33\%), Developer info (2\%) and Using this Widget (0.5\%). For the mobile version, users looked at Description (50\%), Number of users (30\%) and Widget logo (23\%) and ignored Widget status (10\%), Similar Widgets $(9 \%)$ and Developer info $(3 \%)$.

The actual reputation information available remained for the most underutilised, with only the Rating (for website) and Number of users (mobile) among the 'looked

\footnotetext{
${ }^{2}$ http://survey.hiit.fi/index.php?sid=52912\&newtest=Y

${ }^{3}$ One questionnaire respondent did not state gender.
} 
at' elements. Instead, the Description was predominating for both UIs. Developer info was among least attractive elements. A partial reason for this may be how this information is currently shown to the users: behind an additional mouse click.

Trust. Overall, users expressed a trusting attitude towards widget sharing, with $76 \%$ trusting the service "Absolutely" or "Pretty much", and only $5 \%$ "not really" trusting the service. The likelihood of being confronted by harmful content on the WidSets site was considered to be a real risk by as high as $44 \%$ of the users, whereas $46 \%$ stated that this was unlikely. Only $5 \%$ of the users expressed a careless attitude, "not caring" if there is the possibility of downloading harmful content from the site.

\section{Discussion and Conclusions}

The outcomes of our study give evidence of the prominence of the UI visual elements as the source for information behind the downloading - trust - decision. However, the accuracy of the interpretation of these elements and their interplay remain ambiguous. There seems to be little awareness of the underlying security issues related to possible malicious widgets and developers. In the current state with no practical risk this has not been a real issue but new type of widgets may change the situation in the future.

We found users consider the most important elements affecting download decisions to be the description, widget logo (reflecting also 'branding effect') and either rating (in web site) or number of users (in mobile UI). Why rating was found so important in the web UI may be due to its highly visible appearance. Various community features like discussion boards, ratings, comments and presentation of developer history, are hardly used. A friend's network does not appear to be utilized.

The importance of visual appearance suggests that important reputation elements, such as developer reputation, should be presented in a visually prominent manner. An important question is, should the system display the user an aggregation of various reputation data, or should the reputation elements be presented separately, allowing the user to make his or her conclusions in a more independent fashion.

Acknowledgements. This work was supported by TEKES as part of the Future Internet program of the ICT cluster of the Finnish Strategic Centres for Science, Technology and Innovation. The users in the study, and the efforts made by Patrik Floréen, Petteri Nurmi, Yiyun Shen and Sini Ruohomaa in commenting the questionnaire are thankfully acknowledged.

\section{References}

1. Resnick, P., Zeckhauser, R., Swanson, J., Lockwood, K.: The Value of Reputation on eBay: A Controlled Experiment. Experimental Economics 9(2), 79-101 (2006)

2. Riegelsberger, J., Sasse, M.A., McCarthy, J.D.: The mechanics of trust: A framework for research and design. International J. of Human-Computer Studies 62(3), 381-422 (2005) 